International Journal of Pure and Applied Mathematics

Volume 85 No. $4 \quad 2013,691-706$

ISSN: 1311-8080 (printed version); ISSN: 1314-3395 (on-line version)

url: http://www.ijpam.eu

doi: http://dx.doi.org/10.12732/ijpam.v85i4.6

\title{
A GENERAL SOLUTION OF A QUARTIC FUNCTIONAL EQUATION AND ITS STABILITY
}

Charinthip Hengkrawit ${ }^{1}$, Anurak Thanyacharoen ${ }^{2}$

1,2Department of Mathematics and Statistics

Faculty of Science and Technology

Thammasat University

Phatumthani, 12121, THAILAND

Abstract: A general solution of the functional equation

$$
\begin{aligned}
f(x+3 y)+f(x-3 y)+f(x+2 y) & +f(x-2 y)+22 f(x) \\
& =13(f(x+y)+f(x-y))+168 f(y)
\end{aligned}
$$

is determined over the reals without assuming any regularity conditions, and the stability of this functional equation is established.

AMS Subject Classification: 39B22

Key Words: additive function, difference operator, Fréchet functional equation, quartic functional equation

\section{Introduction}

A function $A: \mathbb{R} \rightarrow \mathbb{R}$ is said to be additive, [1], if $A(x+y)=A(x)+A(y)$. For $n \in \mathbb{N}$, a function $A_{n}: \mathbb{R}^{n} \rightarrow \mathbb{R}$ that is additive in each of its variable is said to be $n$-additive. If

$$
A_{n}\left(x_{1}, x_{2}, \ldots, x_{n}\right)=A_{n}\left(x_{\pi(1)}, x_{\pi(2)}, \ldots, x_{\pi(n)}\right)
$$

for every permutation $\{\pi(1), \pi(2), \ldots, \pi(n)\}$ of $\{1,2, \ldots, n\} \quad(n \in \mathbb{N})$, then $A_{n}$ is said to be symmetric. Denote the diagonal element $A_{n}(x, x, \ldots, x)$ by $A^{n}(x)$, if

Received: January 9, 2013

(c) 2013 Academic Publications, Ltd.

$\S$ Correspondence author 
$A_{n}\left(x_{1}, x_{2}, \ldots, x_{n}\right)$ is an $n$-additive symmetric function, and denote the resulted function obtained by putting $x_{1}=x_{2}=\ldots=x_{\ell}=x$ and $x_{\ell+1}=x_{\ell+2}=\ldots=$ $x_{n}=y$ in $A_{n}\left(x_{1}, x_{2}, \ldots, x_{n}\right)$ by $A^{\ell, n-\ell}(x, y)$.

For $f: \mathbb{R} \rightarrow \mathbb{R}$, the difference operator $\Delta_{h}$ with $h \in \mathbb{R}$ is defined by

$$
\Delta_{h} f(x)=f(x+h)-f(x) .
$$

The superposition of difference operations is defined by

$$
\Delta_{h_{1}, \ldots, h_{n}} f=\Delta_{h_{1}} \circ \Delta_{h_{2}} \circ \ldots \circ \Delta_{h_{n}} f, \quad(n \in \mathbb{N}),
$$

where $\Delta_{h_{i}} \circ \Delta_{h_{j}}$ denotes composition. If $h_{1}=h_{2}=\ldots=h_{n}=h$, we write

$$
\Delta_{\underbrace{h, \ldots, h}_{n}} f=\Delta_{h}^{n} f
$$

For $n \in \mathbb{N} \cup\{0\}$, if $f$ satisfies the functional equation

$$
\Delta_{h}^{n+1} f(x)=0 \quad(x, h \in \mathbb{R}),
$$

then $f$ is called a polynomial function of order $n$. In explicit form (1.1) can be written as

$$
\sum_{k=0}^{n+1}(-1)^{n+1-k}\left(\begin{array}{c}
n+1 \\
k
\end{array}\right) f(x+k h)=0 .
$$

It is known ([4]) that with functions defined over $\mathbb{R}$, the equation (1.2) is equivalent to the Fréchet functional equation

$$
\Delta_{h_{1}, \ldots, h_{n+1}} f(x)=0 \quad\left(x, h_{1}, \ldots, h_{n+1} \in \mathbb{R}\right) .
$$

We quote three results from [3, pp.71-77, Theorems 9.3, 9.4, 9.6] which are needed in our work here.

Theorem 1.1. I. If $f: \mathbb{R} \rightarrow \mathbb{R}$ is a polynomial function of order $n$, then

$$
\Delta_{h_{1}, \ldots, h_{n+1}} f(x)=0 \quad\left(x, h_{1}, \ldots, h_{n+1} \in \mathbb{R}\right) .
$$

II. If $A_{k}: \mathbb{R}^{k} \rightarrow \mathbb{R}(k=0,1, \ldots, n)$ are symmetric $k$-additive functions and if $A^{k}$ are their diagonalizations, then the function $\sum_{k=0}^{n} A^{k}(x)$ is a polynomial function of order $n$.

III. If $f: \mathbb{R} \rightarrow \mathbb{R}$ is a polynomial function of order $n$, then there exist $k$-additive symmetric functions $A_{k}: \mathbb{R}^{k} \rightarrow \mathbb{R}(k=0,1, \ldots, n)$ such that $f(x)=$ $\sum_{k=0}^{n} A^{k}(x)$, where $A^{k}$ are the diagonalizations of $A_{k}$. 
Our work here is originated from the functional equation

$$
\begin{array}{r}
f(x+2 y)+f(x-2 y)+6 f(x)=4(f(x+y)+f(x-y)+6 f(y)) \\
(x, y \in \mathbb{R}),
\end{array}
$$

which was considered by Chung and Sahoo, [2] in 2003. Clearly, $f(x)=x^{4}$ is a solution of (1.4), which leads us to call (1.4) a quartic functional equation, and a solution of (1.4) is called a quartic function. Chung and Sahoo's results are :

Theorem 1.2. I. If $f: \mathbb{R} \rightarrow \mathbb{R}$ satisfies the functional equation (1.4), then $f$ is a solution of the Fréchet functional equation

$$
\Delta_{x_{1}, x_{2}, x_{3}, x_{4}, x_{5}} f\left(x_{0}\right)=0 \quad\left(x_{0}, x_{1}, x_{2}, x_{3}, x_{4}, x_{5} \in \mathbb{R}\right) .
$$

II. The function $f: \mathbb{R} \rightarrow \mathbb{R}$ satisfies the functional equation (1.4) if and only if it is of the form $f(x)=A^{4}(x)$, where $A^{4}(x)$ is diagonal of a 4-additive symmetric function $A_{4}$.

Later in 2004, Sahoo, [5], considered the functional equation

$$
f(x+2 y)+f(x-2 y)+6 f(x)=4(f(x+y)+f(x-y)) \quad(x, y \in \mathbb{R}),
$$

and showed that its general solution is of the form

$$
f(x)=A^{0}+A^{1}(x)+A^{2}(x)+A^{3}(x),
$$

where $A^{n}(x)$ is the diagonal of the $n$-additive symmetric function $A_{n}: \mathbb{R}^{n} \rightarrow$ $\mathbb{R}(n=1,2,3)$ and $A^{0}$ is an arbitrary constant. One year later, he generalized (1.5) to

$$
f_{1}(2 x+y)+f_{2}(2 x-y)=f_{3}(x+y)+f_{4}(x-y)+f_{5}(x) \quad(x, y \in \mathbb{R}),
$$

and proved that $f_{1}, f_{2}, f_{3}, f_{4}, f_{5}: \mathbb{R} \rightarrow \mathbb{R}$ satisfy (1.6) if and only if

$$
\begin{aligned}
f_{1}(x) & =A^{3}(x)+A^{2}(x)+A^{1}(x)+A^{0}+B^{2}(x)+B^{1}(x)+B^{0}, \\
f_{2}(x) & =A^{3}(x)+A^{2}(x)+A^{1}(x)+A^{0}-B^{2}(x)-B^{1}(x)-B^{0}, \\
f_{3}(x) & =2 A^{3}(x)+A^{2}(x)+A^{1}(x)+\frac{1}{2} A^{0}+C^{1}(x) \\
& +C^{0}+2 B^{2}(x)+B^{1}(x)+B^{0}+D^{0}, \\
f_{4}(x) & =2 A^{3}(x)+A^{2}(x)+A^{1}(x)+\frac{1}{2} A^{0}+C^{1}(x) \\
& +C^{0}-2 B^{2}(x)-B^{1}(x)-B^{0}-D^{0},
\end{aligned}
$$




$$
f_{5}(x)=12 A^{3}(x)+6 A^{2}(x)+2 A^{1}(x)+A^{0}-2 C^{1}(x)-2 C^{0},
$$

where $A^{0}, B^{0}, C^{0}, D^{0}$ are arbitrary constants, $A^{n}(x), B^{n}(x), C^{n}(x)$ are the diagonals of $n$-additive symmetric functions $A_{n}, B_{n}, C_{n}$, respectively.

Recognizing the identity

$$
\begin{aligned}
(x+3 y)^{4}+(x-3 y)^{4} & +(x+2 y)^{4}+(x-2 y)^{4}+22 x^{4} \\
& =13\left((x+y)^{4}+(x-y)^{4}\right)+168 y^{4}
\end{aligned}
$$

which renders a solution $f(x)=x^{4}$ to the functional equation

$$
\begin{aligned}
f(x+3 y)+f(x-3 y) & +f(x+2 y)+f(x-2 y)+22 f(x) \\
& =13(f(x+y)+f(x-y))+168 f(y),
\end{aligned}
$$

here we find a general solution of (1.9) without assuming any regularity condition and establish its stabilitya stability. Our main results are:

Theorem 1.3. I. A function $f: \mathbb{R} \rightarrow \mathbb{R}$ satisfies the functional equation (1.9) if and only if it is of the form

$$
f(x)=A^{4}(x) \quad(x \in \mathbb{R}),
$$

where $A^{4}(x)$ is the diagonal of a 4-additive symmetric function $A_{4}$. II. Let $\phi: \mathbb{R}^{2} \rightarrow[0, \infty)$ be a function such that the series

$$
\sum_{i=0}^{\infty} \frac{\phi\left(2^{i} x, 2^{i} y\right)}{16^{i}}
$$

converges for all $x, y \in \mathbb{R}$. If $f: \mathbb{R} \rightarrow \mathbb{R}$ is an even function with $f(0)=0$ and satisfies

$$
|D f(x, y)| \leq \phi(x, y)
$$

where

$$
\begin{aligned}
D f(x, y):=f(x+3 y)+f(x-3 y)+f(x+2 y)+f(x-2 y) & +22 f(x) \\
& -13 f(x+y)
\end{aligned}
$$

then there exists a unique $T: \mathbb{R} \rightarrow \mathbb{R}$ satisfying (1.9) and

$$
|f(y)-T(y)| \leq \frac{1}{5376} \times
$$




$$
\sum_{i=0}^{\infty} \frac{2 \phi\left(3 \cdot 2^{i} y, 2^{i} y\right)+2 \phi\left(2 \cdot 2^{i} y, 2^{i} y\right)+\phi\left(0,2 \cdot 2^{i} y\right)+30 \phi\left(2^{i} y, 2^{i} y\right)+50 \phi\left(0,2^{i} y\right)}{16^{i}}
$$

where $y \in \mathbb{R}$. Moreover, the function $T$ is given by

$$
T(y)=\lim _{n \rightarrow \infty} \frac{f\left(2^{n} y\right)}{16^{n}} \quad(y \in \mathbb{R}) .
$$

\section{Proof of Theorem $1.3 \mathrm{I}$}

In this section, we establish the general solution of the functional equation (1.9). First, we prove an auxiliary lemma.

Lemma 2.1. If $f: \mathbb{R} \rightarrow \mathbb{R}$ satisfies the functional equation

$$
f(x+4 y)-14 f(x+2 y)+35 f(x+y)-35 f(x)+14 f(x-y)-f(x-3 y)=0,
$$

where $x, y \in \mathbb{R}$, then $f$ is a solution of the Fréchet functional equation

$$
\Delta_{x_{1}, x_{2}, x_{3}, x_{4}, x_{5}, f} f\left(x_{0}\right)=0 \quad\left(x_{0}, x_{1}, x_{2}, x_{3}, x_{4}, x_{5} \in \mathbb{R}\right) .
$$

Proof. Substituting

$$
x_{0}=x+4 y, \quad y_{1}=x-3 y, \text { i.e., } x=\frac{3}{7} x_{0}+\frac{4}{7} y_{1}, y=\frac{1}{7} x_{0}-\frac{1}{7} y_{1}
$$

in $(2.1)$, we obtain

$$
\begin{aligned}
f\left(x_{0}\right)-14 f\left(\frac{5}{7} x_{0}+\frac{2}{7} y_{1}\right) & +35 f\left(\frac{4}{7} x_{0}+\frac{3}{7} y_{1}\right)-35 f\left(\frac{3}{7} x_{0}+\frac{4}{7} y_{1}\right) \\
& +14 f\left(\frac{2}{7} x_{0}+\frac{5}{7} y_{1}\right)-f\left(y_{1}\right)=0 .
\end{aligned}
$$

Replacing $x_{0}$ by $x_{0}+x_{1}$ in (2.2) and subtracting (2.2) from the resulting expression, we obtain

$$
\begin{aligned}
& f\left(x_{0}+x_{1}\right)-f\left(x_{0}\right)-14 f\left(\frac{5}{7}\left(x_{0}+x_{1}\right)+\frac{2}{7} y_{1}\right)+14 f\left(\frac{5}{7} x_{0}+\frac{2}{7} y_{1}\right) \\
& +35 f\left(\frac{4}{7}\left(x_{0}+x_{1}\right)+\frac{3}{7} y_{1}\right)-35 f\left(\frac{4}{7} x_{0}+\frac{3}{7} y_{1}\right)-35 f\left(\frac{3}{7}\left(x_{0}+x_{1}\right)+\frac{4}{7} y_{1}\right)
\end{aligned}
$$




$$
+35 f\left(\frac{3}{7} x_{0}+\frac{4}{7} y_{1}\right)+14 f\left(\frac{2}{7}\left(x_{0}+x_{1}\right)+\frac{5}{7} y_{1}\right)-14 f\left(\frac{2}{7} x_{0}+\frac{5}{7} y_{1}\right)=0
$$

Putting $y_{2}=\frac{4}{7} x_{0}+\frac{3}{7} y_{1}$, i.e., $y_{1}=-\frac{4}{3} x_{0}+\frac{7}{3} y_{2}$ in $(2.4)$, we see that

$$
\begin{aligned}
& f\left(x_{0}+x_{1}\right)-f\left(x_{0}\right)-14 f\left(\frac{5}{7} x_{1}+\frac{1}{3} x_{0}+\frac{2}{3} y_{2}\right)+14 f\left(\frac{1}{3} x_{0}+\frac{2}{3} y_{2}\right) \\
& +35 f\left(\frac{4}{7} x_{1}+y_{2}\right)-35 f\left(y_{2}\right)-35 f\left(\frac{3}{7} x_{1}-\frac{1}{3} x_{0}+\frac{4}{3} y_{2}\right) \\
& +35 f\left(-\frac{1}{3} x_{0}+\frac{4}{3} y_{2}\right)+14 f\left(\frac{2}{7} x_{1}-\frac{2}{3} x_{0}+\frac{5}{3} y_{2}\right)-14 f\left(-\frac{2}{3} x_{0}+\frac{5}{3} y_{2}\right)=0 .
\end{aligned}
$$

Replacing $x_{0}$ by $x_{0}+x_{2}$ in (2.5) and subtracting (2.5) from the resulting expression, we obtain

$$
\begin{aligned}
& f\left(x_{0}+x_{1}+x_{2}\right)-f\left(x_{0}+x_{1}\right)-f\left(x_{0}+x_{2}\right)+f\left(x_{0}\right) \\
& -14 f\left(\frac{5}{7} x_{1}+\frac{1}{3}\left(x_{0}+x_{2}\right)+\frac{2}{3} y_{2}\right)+14 f\left(\frac{5}{7} x_{1}+\frac{1}{3} x_{0}+\frac{2}{3} y_{2}\right) \\
& +14 f\left(\frac{1}{3}\left(x_{0}+x_{2}\right)+\frac{2}{3} y_{2}\right)-14 f\left(\frac{1}{3} x_{0}+\frac{2}{3} y_{2}\right) \\
& -35 f\left(\frac{3}{7} x_{1}-\frac{1}{3}\left(x_{0}+x_{2}\right)+\frac{4}{3} y_{2}\right)+35 f\left(\frac{3}{7} x_{1}-\frac{1}{3} x_{0}+\frac{4}{3} y_{2}\right) \\
& +35 f\left(-\frac{1}{3}\left(x_{0}+x_{2}\right)+\frac{4}{3} y_{2}\right)-35 f\left(-\frac{1}{3} x_{0}+\frac{4}{3} y_{2}\right) \\
& +14 f\left(\frac{2}{7} x_{1}-\frac{2}{3}\left(x_{0}+x_{2}\right)+\frac{5}{3} y_{2}\right)-14 f\left(\frac{2}{7} x_{1}-\frac{2}{3} x_{0}+\frac{5}{3} y_{2}\right) \\
& -14 f\left(-\frac{2}{3}\left(x_{0}+x_{2}\right)+\frac{5}{3} y_{2}\right)+14 f\left(-\frac{2}{3} x_{0}+\frac{5}{3} y_{2}\right)=0 .
\end{aligned}
$$

Letting $y_{3}=\frac{1}{3} x_{0}+\frac{2}{3} y_{2}$, i.e., $y_{2}=-\frac{1}{2} x_{0}+\frac{3}{2} y_{3}$ in $(2.6)$, we see that

$$
\begin{aligned}
& f\left(x_{0}+x_{1}+x_{2}\right)-f\left(x_{0}+x_{1}\right)-f\left(x_{0}+x_{2}\right)+f\left(x_{0}\right) \\
& -14 f\left(\frac{5}{7} x_{1}+\frac{1}{3} x_{2}+y_{3}\right)+14 f\left(\frac{5}{7} x_{1}+y_{3}\right) \\
& +14 f\left(\frac{1}{3} x_{2}+y_{3}\right)-14 f\left(y_{3}\right)-35 f\left(\frac{3}{7} x_{1}-\frac{1}{3} x_{2}-x_{0}+2 y_{3}\right) \\
& +35 f\left(\frac{3}{7} x_{1}-x_{0}+2 y_{3}\right)+35 f\left(-\frac{1}{3} x_{2}-x_{0}+2 y_{3}\right)-35 f\left(-x_{0}+2 y_{3}\right)
\end{aligned}
$$




$$
\begin{aligned}
& +14 f\left(\frac{2}{7} x_{1}-\frac{2}{3} x_{2}-\frac{3}{2} x_{0}+\frac{5}{2} y_{3}\right)-14 f\left(\frac{2}{7} x_{1}-\frac{3}{2} x_{0}+\frac{5}{2} y_{3}\right) \\
& -14 f\left(-\frac{2}{3} x_{2}-\frac{3}{2} x_{0}+\frac{5}{2} y_{3}\right)+14 f\left(-\frac{3}{2} x_{0}+\frac{5}{2} y_{3}\right)=0 .
\end{aligned}
$$

Replacing $x_{0}$ by $x_{0}+x_{3}$ in (2.7) and subtracting (2.7) from the resulting expression, we obtain

$$
\begin{aligned}
& f\left(x_{0}+x_{1}+x_{2}+x_{3}\right)-f\left(x_{0}+x_{1}+x_{2}\right)-f\left(x_{0}+x_{1}+x_{3}\right)-f\left(x_{0}+x_{2}+x_{3}\right) \\
& +f\left(x_{0}+x_{1}\right)+f\left(x_{0}+x_{2}\right)+f\left(x_{0}+x_{3}\right)-f\left(x_{0}\right) \\
& -35 f\left(\frac{3}{7} x_{1}-\frac{1}{3} x_{2}-\left(x_{0}+x_{3}\right)+2 y_{3}\right)+35 f\left(\frac{3}{7} x_{1}-\frac{1}{3} x_{2}-x_{0}+2 y_{3}\right) \\
& +35 f\left(\frac{3}{7} x_{1}-\left(x_{0}+x_{3}\right)+2 y_{3}\right)-35 f\left(\frac{3}{7} x_{1}-x_{0}+2 y_{3}\right) \\
& +35 f\left(-\frac{1}{3} x_{2}-\left(x_{0}+x_{3}\right)+2 y_{3}\right)-35 f\left(-\frac{1}{3} x_{2}-x_{0}+2 y_{3}\right) \\
& -35 f\left(-\left(x_{0}+x_{3}\right)+2 y_{3}\right)+35 f\left(-x_{0}+2 y_{3}\right) \\
& +14 f\left(\frac{2}{7} x_{1}-\frac{2}{3} x_{2}-\frac{3}{2}\left(x_{0}+x_{3}\right)+\frac{5}{2} y_{3}\right)-14 f\left(\frac{2}{7} x_{1}-\frac{2}{3} x_{2}-\frac{3}{2} x_{0}+\frac{5}{2} y_{3}\right) \\
& -14 f\left(\frac{2}{7} x_{1}-\frac{3}{2}\left(x_{0}+x_{3}\right)+\frac{5}{2} y_{3}\right)+14 f\left(\frac{2}{7} x_{1}-\frac{3}{2} x_{0}+\frac{5}{2} y_{3}\right) \\
& -14 f\left(-\frac{2}{3} x_{2}-\frac{3}{2}\left(x_{0}+x_{3}\right)+\frac{5}{2} y_{3}\right)+14 f\left(-\frac{2}{3} x_{2}-\frac{3}{2} x_{0}+\frac{5}{2} y_{3}\right) \\
& +14 f\left(-\frac{3}{2}\left(x_{0}+x_{3}\right)+\frac{5}{2} y_{3}\right)-14 f\left(-\frac{3}{2} x_{0}+\frac{5}{2} y_{3}\right)=0 . \\
& +14
\end{aligned}
$$

Putting $y_{4}=-x_{0}+2 y_{3}$, i.e., $y_{3}=\frac{1}{2} x_{0}+\frac{1}{2} y_{4}$ in $(2.8)$, we see that

$$
\begin{aligned}
& f\left(x_{0}+x_{1}+x_{2}+x_{3}\right)-f\left(x_{0}+x_{1}+x_{2}\right)-f\left(x_{0}+x_{1}+x_{3}\right)-f\left(x_{0}+x_{2}+x_{3}\right) \\
& +f\left(x_{0}+x_{1}\right)+f\left(x_{0}+x_{2}\right)+f\left(x_{0}+x_{3}\right)-f\left(x_{0}\right) \\
& -35 f\left(\frac{3}{7} x_{1}-\frac{1}{3} x_{2}-x_{3}+y_{4}\right) \\
& +35 f\left(\frac{3}{7} x_{1}-\frac{1}{3} x_{2}+y_{4}\right)+35 f\left(\frac{3}{7} x_{1}-x_{3}+y_{4}\right)-35 f\left(\frac{3}{7} x_{1}+y_{4}\right)
\end{aligned}
$$




$$
\begin{aligned}
& +35 f\left(-\frac{1}{3} x_{2}-x_{3}+y_{4}\right)-35 f\left(-\frac{1}{3} x_{2}+y_{4}\right)-35 f\left(-x_{3}+y_{4}\right)+35 f\left(y_{4}\right) \\
& +14 f\left(\frac{2}{7} x_{1}-\frac{2}{3} x_{2}-\frac{3}{2} x_{3}-\frac{1}{4} x_{0}+\frac{5}{4} y_{4}\right)-14 f\left(\frac{2}{7} x_{1}-\frac{2}{3} x_{2}-\frac{1}{4} x_{0}+\frac{5}{4} y_{4}\right) \\
& -14 f\left(\frac{2}{7} x_{1}-\frac{3}{2} x_{3}-\frac{1}{4} x_{0}+\frac{5}{4} y_{4}\right)+14 f\left(\frac{2}{7} x_{1}-\frac{1}{4} x_{0}+\frac{5}{4} y_{4}\right) \\
& -14 f\left(-\frac{2}{3} x_{2}-\frac{3}{2} x_{3}-\frac{1}{4} x_{0}+\frac{5}{4} y_{4}\right)+14 f\left(-\frac{2}{3} x_{2}-\frac{1}{4} x_{0}+\frac{5}{4} y_{4}\right) \\
& +14 f\left(-\frac{3}{2} x_{3}-\frac{1}{4} x_{0}+\frac{5}{4} y_{4}\right)-14 f\left(-\frac{1}{4} x_{0}+\frac{5}{4} y_{4}\right)=0 .
\end{aligned}
$$

Replacing $x_{0}$ by $x_{0}+x_{4}$ in (2.9) and subtracting (2.9) from the resulting expression, we obtain

$$
\begin{aligned}
& f\left(x_{0}+x_{1}+x_{2}+x_{3}+x_{4}\right)-f\left(x_{0}+x_{1}+x_{2}+x_{3}\right) \\
& -f\left(x_{0}+x_{1}+x_{2}+x_{4}\right) \\
& -f\left(x_{0}+x_{1}+x_{3}+x_{4}\right)-f\left(x_{0}+x_{2}+x_{3}+x_{4}\right)+f\left(x_{0}+x_{1}+x_{2}\right) \\
& +f\left(x_{0}+x_{1}+x_{3}\right)+f\left(x_{0}+x_{1}+x_{4}\right)+f\left(x_{0}+x_{2}+x_{3}\right)+f\left(x_{0}+x_{2}+x_{4}\right) \\
& +f\left(x_{0}+x_{3}+x_{4}\right)-f\left(x_{0}+x_{1}\right)-f\left(x_{0}+x_{2}\right)-f\left(x_{0}+x_{3}\right)-f\left(x_{0}+x_{4}\right) \\
& +f\left(x_{0}\right)+14 f\left(\frac{2}{7} x_{1}-\frac{2}{3} x_{2}-\frac{3}{2} x_{3}-\frac{1}{4}\left(x_{0}+x_{4}\right)+\frac{5}{4} y_{4}\right) \\
& -14 f\left(\frac{2}{7} x_{1}-\frac{2}{3} x_{2}-\frac{3}{2} x_{3}-\frac{1}{4} x_{0}+\frac{5}{4} y_{4}\right) \\
& -14 f\left(\frac{2}{7} x_{1}-\frac{2}{3} x_{2}-\frac{1}{4}\left(x_{0}+x_{4}\right)+\frac{5}{4} y_{4}\right)+14 f\left(\frac{2}{7} x_{1}-\frac{2}{3} x_{2}-\frac{1}{4} x_{0}+\frac{5}{4} y_{4}\right) \\
& -14 f\left(\frac{2}{7} x_{1}-\frac{3}{2} x_{3}-\frac{1}{4}\left(x_{0}+x_{4}\right)+\frac{5}{4} y_{4}\right)+14 f\left(\frac{2}{7} x_{1}-\frac{3}{2} x_{3}-\frac{1}{4} x_{0}+\frac{5}{4} y_{4}\right) \\
& +14 f\left(\frac{2}{7} x_{1}-\frac{1}{4}\left(x_{0}+x_{4}\right)+\frac{5}{4} y_{4}\right)-14 f\left(\frac{2}{7} x_{1}-\frac{1}{4} x_{0}+\frac{5}{4} y_{4}\right) \\
& -14 f\left(-\frac{2}{3} x_{2}-\frac{3}{2} x_{3}-\frac{1}{4}\left(x_{0}+x_{4}\right)+\frac{5}{4} y_{4}\right)+14 f\left(-\frac{2}{3} x_{2}-\frac{3}{2} x_{3}-\frac{1}{4} x_{0}+\frac{5}{4} y_{4}\right) \\
& +14 f\left(-\frac{2}{3} x_{2}-\frac{1}{4}\left(x_{0}+x_{4}\right)+\frac{5}{4} y_{4}\right)-14 f\left(-\frac{2}{3} x_{2}-\frac{1}{4} x_{0}+\frac{5}{4} y_{4}\right) \\
& +14 f\left(-\frac{3}{2} x_{3}-\frac{1}{4}\left(x_{0}+x_{4}\right)+\frac{5}{4} y_{4}\right)-14 f\left(-\frac{3}{2} x_{3}-\frac{1}{4} x_{0}+\frac{5}{4} y_{4}\right)
\end{aligned}
$$




$$
-14 f\left(-\frac{1}{4}\left(x_{0}+x_{4}\right)+\frac{5}{4} y_{4}\right)+14 f\left(-\frac{1}{4} x_{0}+\frac{5}{4} y_{4}\right)=0 .
$$

Letting $y_{5}=-\frac{1}{4} x_{0}+\frac{5}{4} y_{4}$ in $(2.10)$, we see that

$$
\begin{aligned}
& f\left(x_{0}+x_{1}+x_{2}+x_{3}+x_{4}\right)-f\left(x_{0}+x_{1}+x_{2}+x_{3}\right)-f\left(x_{0}+x_{1}+x_{2}+x_{4}\right) \\
& -f\left(x_{0}+x_{1}+x_{3}+x_{4}\right)-f\left(x_{0}+x_{2}+x_{3}+x_{4}\right)+f\left(x_{0}+x_{1}+x_{2}\right) \\
& +f\left(x_{0}+x_{1}+x_{3}\right)+f\left(x_{0}+x_{1}+x_{4}\right)+f\left(x_{0}+x_{2}+x_{3}\right)+f\left(x_{0}+x_{2}+x_{4}\right) \\
& +f\left(x_{0}+x_{3}+x_{4}\right)-f\left(x_{0}+x_{1}\right)-f\left(x_{0}+x_{2}\right)-f\left(x_{0}+x_{3}\right) \\
& -f\left(x_{0}+x_{4}\right)+f\left(x_{0}\right)+14 f\left(\frac{2}{7} x_{1}-\frac{2}{3} x_{2}-\frac{3}{2} x_{3}-\frac{1}{4} x_{4}+y_{5}\right) \\
& -14 f\left(\frac{2}{7} x_{1}-\frac{2}{3} x_{2}-\frac{3}{2} x_{3}+y_{5}\right)-14 f\left(\frac{2}{7} x_{1}-\frac{2}{3} x_{2}-\frac{1}{4} x_{4}+y_{5}\right) \\
& +14 f\left(\frac{2}{7} x_{1}-\frac{2}{3} x_{2}+y_{5}\right)-14 f\left(\frac{2}{7} x_{1}-\frac{3}{2} x_{3}-\frac{1}{4} x_{4}+y_{5}\right) \\
& +14 f\left(\frac{2}{7} x_{1}-\frac{3}{2} x_{3}+y_{5}\right)+14 f\left(\frac{2}{7} x_{1}-\frac{1}{4} x_{4}+y_{5}\right) \\
& -14 f\left(\frac{2}{7} x_{1}+y_{5}\right)-14 f\left(-\frac{2}{3} x_{2}-\frac{3}{2} x_{3}-\frac{1}{4} x_{4}+y_{5}\right) \\
& +14 f\left(-\frac{2}{3} x_{2}-\frac{3}{2} x_{3}+y_{5}\right)+14 f\left(-\frac{2}{3} x_{2}-\frac{1}{4} x_{4}+y_{5}\right) \\
& -14 f\left(-\frac{2}{3} x_{2}+y_{5}\right)+14 f\left(-\frac{3}{2} x_{3}-\frac{1}{4} x_{4}+y_{5}\right) \\
& -14 f\left(-\frac{3}{2} x_{3}+y_{5}\right)-14 f\left(-\frac{1}{4} x_{4}+y_{5}\right)+14 f\left(y_{5}\right)=0 \text {. }
\end{aligned}
$$

Replacing $x_{0}$ by $x_{0}+x_{5}$ in (2.11) and subtracting (2.11) from the resulting expression, we obtain

$$
\begin{aligned}
& f\left(x_{0}+x_{1}+x_{2}+x_{3}+x_{4}+x_{5}\right)-f\left(x_{0}+x_{1}+x_{2}+x_{3}+x_{4}\right) \\
& -f\left(x_{0}+x_{1}+x_{2}+x_{3}+x_{5}\right)-f\left(x_{0}+x_{1}+x_{2}+x_{4}+x_{5}\right) \\
& -f\left(x_{0}+x_{1}+x_{3}+x_{4}+x_{5}\right)-f\left(x_{0}+x_{2}+x_{3}+x_{4}+x_{5}\right) \\
& +f\left(x_{0}+x_{1}+x_{2}+x_{3}\right)+f\left(x_{0}+x_{1}+x_{2}+x_{4}\right)+f\left(x_{0}+x_{1}+x_{2}+x_{5}\right) \\
& +f\left(x_{0}+x_{1}+x_{3}+x_{4}\right)+f\left(x_{0}+x_{1}+x_{3}+x_{5}\right)+f\left(x_{0}+x_{1}+x_{4}+x_{5}\right) \\
& +f\left(x_{0}+x_{2}+x_{3}+x_{4}\right)+f\left(x_{0}+x_{2}+x_{3}+x_{5}\right)+f\left(x_{0}+x_{2}+x_{4}+x_{5}\right)
\end{aligned}
$$


$+f\left(x_{0}+x_{3}+x_{4}+x_{5}\right)-f\left(x_{0}+x_{1}+x_{2}\right)-f\left(x_{0}+x_{1}+x_{3}\right)-f\left(x_{0}+x_{1}+x_{4}\right)$

$-f\left(x_{0}+x_{1}+x_{5}\right)-f\left(x_{0}+x_{2}+x_{3}\right)-f\left(x_{0}+x_{2}+x_{4}\right)-f\left(x_{0}+x_{2}+x_{5}\right)$

$-f\left(x_{0}+x_{3}+x_{4}\right)-f\left(x_{0}+x_{3}+x_{5}\right)-f\left(x_{0}+x_{4}+x_{5}\right)+f\left(x_{0}+x_{1}\right)$

$+f\left(x_{0}+x_{2}\right)+f\left(x_{0}+x_{3}\right)+f\left(x_{0}+x_{4}\right)+f\left(x_{0}+x_{5}\right)-f\left(x_{0}\right)=0$.

The desired result follows by noting that the left-hand side is indeed $\Delta_{x_{1}, \ldots, x_{5}}$ $f\left(x_{0}\right)$.

We are now ready to prove Theorem $1.3 \mathrm{I}$.

Replacing $x$ by $x+y$ in (1.9), and subtracting (1.9) from the resulting expression, we obtain

$f(x+4 y)-14 f(x+2 y)+35 f(x+y)-35 f(x)+14 f(x-y)-f(x-3 y)=0$,

which by Lemma 2.1 yields $\Delta_{x_{1}, \ldots, x_{5}} f\left(x_{0}\right)=0$. Invoking upon Theorem 1.1 III, we deduce that

$$
f(x)=A^{4}(x)+A^{3}(x)+A^{2}(x)+A^{1}(x)+A^{0} \quad(x \in \mathbb{R}) .
$$

Now, replacing $y$ by $-y$ in (1.9), we get

$$
\begin{aligned}
f(x+3 y)+f(x-3 y)+f(x+2 y) & +f(x-2 y)+22 f(x) \\
& =13(f(x+y)+f(x-y))+168 f(-y) .
\end{aligned}
$$

Subtracting (2.14) from (1.9), we get $f(y)=f(-y)$, i.e., $f$ is even, which forces $A^{3}(x)$ and $A^{1}(x)$ to vanish. Since $f(0)=0$, we have $A^{0} \equiv 0$. Putting these into (2.13), we have

$$
f(x)=A^{4}(x)+A^{2}(x) .
$$

Substituting $f$ from (2.16) into (1.9), we obtain

$$
\begin{aligned}
& A^{4}(x+3 y)+A^{4}(x-3 y)+A^{4}(x+2 y)+A^{4}(x-2 y)+A^{2}(x+3 y) \\
& \quad+A^{2}(x-3 y)+A^{2}(x+2 y)+A^{2}(x-2 y)+22\left(A^{4}(x)+A^{2}(x)\right) \\
& =13\left(A^{4}(x+y)+A^{4}(x-y)+A^{2}(x+y)+A^{2}(x-y)\right) \\
& \quad+168\left(A^{4}(y)+A^{2}(y)\right) .
\end{aligned}
$$

Using

$$
A^{4}(x+y)+A^{4}(x-y)=2 A^{4}(x)+12 A^{2,2}(x, y)+2 A^{4}(y)
$$




$$
\begin{aligned}
A^{2}(x+y)+A^{2}(x-y) & =2 A^{2}(x)+2 A^{2}(y) \\
A^{2,2}(x, 3 y) & =9 A^{2,2}(x, y) \\
A^{4}(3 y) & =81 A^{4}(y) \\
A^{2,2}(x, 2 y) & =4 A^{2,2}(x, y) \\
A^{4}(2 y) & =16 A^{4}(y) \\
A^{2}(2 y) & =4 A^{2}(y) \\
A^{2}(3 y) & =9 A^{2}(y),
\end{aligned}
$$

we get

$$
\begin{aligned}
& 2 A^{4}(x)+108 A^{2,2}(x, y)+162 A^{4}(y)+2 A^{4}(x)+48 A^{2,2}(x, y)+32 A^{4}(y) \\
&+2 A^{2}(x)+18 A^{2}(y)+2 A^{2}(x)+8 A^{2}(y)+22\left(A^{4}(x)+A^{2}(x)\right) \\
&= 13\left(2 A^{4}(x)+12 A^{2,2}(x, y)+2 A^{4}(y)+2 A^{2}(x)+2 A^{2}(y)\right) \\
&+168\left(A^{4}(y)+A^{2}(y)\right),
\end{aligned}
$$

which simplifies to $A^{2}(y)=0$, and so (2.16) yields $f(x)=A^{4}(x)$.

Conversely, assume that there exists a 4-additive symmetric function $A_{4}$ : $\mathbb{R}^{4} \rightarrow \mathbb{R}$ such that $f(x)=A^{4}(x)$. Since

$$
\begin{aligned}
A^{4}(x+y)+A^{4}(x-y) & =2 A^{4}(x)+12 A^{2,2}(x, y)+2 A^{4}(y), \\
A^{2,2}(x, 2 y) & =4 A^{2,2}(x, y), A^{4}(2 y)=16 A^{4}(y),
\end{aligned}
$$

we have

$$
\begin{aligned}
f(x+3 y)+f(x-3 y)+f(x+2 y)+f(x-2 y)+22 f(x) \\
=A^{4}(x+3 y)+A^{4}(x-3 y)+A^{4}(x+2 y)+A^{4}(x-2 y)+22 A^{4}(x) \\
=2 A^{4}(x)+108 A^{2,2}(x, y)+162 A^{4}(y)+2 A^{4}(x)+48 A^{2,2}(x, y) \\
\quad+32 A^{4}(y)+22 A^{4}(x) \\
=26 A^{4}(x)+156 A^{2,2}(x, y)+194 A^{4}(y) \\
=13\left(2 A^{4}(x)+12 A^{2,2}(x, y)+2 A^{4}(y)\right)+168 A^{4}(y) \\
=13(f(x+y)+f(x-y))+168 f(y) .
\end{aligned}
$$

\section{Proof of Theorem 1.3 II}

We first prove an auxialiary result. 
Lemma 3.1. If $T: \mathbb{R} \rightarrow \mathbb{R}$ satisfies (1.9), then $T\left(2^{n} y\right)=16^{n} T(y)$ for each $n \in \mathbb{N}$.

Proof. The case $n=1$ follows at once from Theorem 1.3 I, namely,

$$
T(2 y)=A^{4}(2 y)=16 A^{4}(y)=16 T(y)
$$

Assume the assertion holds up to $k$. Since $A^{4}(y)$ is the diagonal of a 4 -additive symmetric function $A_{4}: \mathbb{R}^{4} \rightarrow \mathbb{R}$ and $T(y)=A^{4}(y)$, using induction we have

$$
\begin{aligned}
T\left(2^{k+1} y\right) & =A^{4}\left(2^{k} y+2^{k} y\right)=A_{4}\left(2^{k} y+2^{k} y, 2^{k} y+2^{k} y, 2^{k} y+2^{k} y, 2^{k} y+2^{k} y\right) \\
& =16 A_{4}\left(2^{k} y, 2^{k} y, 2^{k} y, 2^{k} y\right)=16 A^{4}\left(2^{k} y\right)=16 T\left(2^{k} y\right)=16^{k+1} T(y) .
\end{aligned}
$$

Now we proceed to prove theorem 1.3 II. Putting $x=3 y$ in (1.13) and using $f(0)=0$, we have

$$
|f(6 y)+f(5 y)-13 f(4 y)+22 f(3 y)-13 f(2 y)-167 f(y)| \leq \phi(3 y, y) .
$$

Putting $x=2 y$ in (1.13), using $f(0)=0$ and $f$ is even, we have

$$
|f(5 y)+f(4 y)-13 f(3 y)+22 f(2 y)-180 f(y)| \leq \phi(2 y, y) .
$$

From (3.1) and (3.2), we obtain

$$
|f(6 y)-14 f(4 y)+35 f(3 y)-35 f(2 y)+13 f(y)| \leq \phi(3 y, y)+\phi(2 y, y) .
$$

Putting $x=0$ in (1.13), using $f(0)=0$ and $f$ is even, we have

$$
|f(3 y)+f(2 y)-97 f(y)| \leq \frac{\phi(0, y)}{2}
$$

Replacing $y$ by $2 y$ in (3.4). we obtain

$$
|f(6 y)+f(4 y)-97 f(2 y)| \leq \frac{\phi(0,2 y)}{2} .
$$

From (3.3) and (3.5), we obtain

$$
|-15 f(4 y)+35 f(3 y)+62 f(2 y)+13 f(y)| \leq \phi(3 y, y)+\phi(2 y, y)+\frac{\phi(0,2 y)}{2} \text {. }
$$


Putting $x=y$ in (1.13), using $f(0)=0$ and $f$ is even, we have

$$
|15 f(4 y)+15 f(3 y)-180 f(2 y)-2175 f(y)| \leq 15 \phi(y, y) .
$$

From (3.6) and (3.7), we obtain

$$
|50 f(3 y)-118 f(2 y)-2162 f(y)| \leq \phi(3 y, y)+\phi(2 y, y)+\frac{\phi(0,2 y)}{2}+15 \phi(y, y)
$$

From (3.4), we have

$$
|50 f(3 y)+50 f(2 y)-4850 f(y)| \leq 25 \phi(0, y)
$$

From (3.8) and (3.9), we obtain

$$
|f(2 y)-16 f(y)| \leq \frac{1}{168}\left(\phi(3 y, y)+\phi(2 y, y)+\frac{\phi(0,2 y)}{2}+15 \phi(y, y)+25 \phi(0, y)\right) \text {. }
$$

Dividing by 16 in (3.10), we have

$$
\left|\frac{f(2 y)}{16}-f(y)\right| \leq \frac{1}{5376}[2 \phi(3 y, y)+2 \phi(2 y, y)+\phi(0,2 y)+30 \phi(y, y)+50 \phi(0, y)] \text {. }
$$

Replacing $y$ by $2 y$ in (3.11) and dividing by 16 , we obtain

$$
\begin{aligned}
& \left|\frac{f\left(2^{2} y\right)}{(16)^{2}}-\frac{f(2 y)}{16}\right| \\
& \leq \frac{1}{5376}\left[\frac{2 \phi(3(2 y), 2 y)+2 \phi(2(2 y), 2 y)+\phi(0,2(2 y))+30 \phi(2 y, 2 y)+50 \phi(0,2 y)}{16}\right] .
\end{aligned}
$$

From the equations (3.11) and (3.12), we have

$$
\begin{aligned}
& \left|\frac{f\left(2^{2} y\right)}{(16)^{2}}-f(y)\right| \\
& \leq \frac{1}{5376}\left(\frac{2 \phi(3(2 y), 2 y)+2 \phi(2(2 y), 2 y)+\phi(0,2(2 y))+30 \phi(2 y, 2 y)+50 \phi(0,2 y)}{16}\right. \\
& \quad+2 \phi(3 y, y)+2 \phi(2 y, y)+\phi(0,2 y)+30 \phi(y, y)+50 \phi(0, y))
\end{aligned}
$$


By induction, (3.13) extends to

$$
\begin{aligned}
& \left|\frac{f\left(2^{n} y\right)}{16^{n}}-f(y)\right| \\
& \leq \frac{1}{5376} \sum_{i=0}^{n-1} \frac{2 \phi\left(3\left(2^{i} y\right), 2^{i} y\right)+2 \phi\left(2\left(2^{i} y\right), 2^{i} y\right)+\phi\left(0,2\left(2^{i} y\right)\right)+30 \phi\left(2^{i} y, 2^{i} y\right)+50 \phi\left(o, 2^{i} y\right)}{16^{i}} \\
& \leq \frac{1}{5376} \sum_{i=0}^{\infty} \frac{2 \phi\left(3\left(2^{i} y\right), 2^{i} y\right)+2 \phi\left(2\left(2^{i} y\right), 2^{i} y\right)+\phi\left(0,2\left(2^{i} y\right)\right)+30 \phi\left(2^{i} y, 2^{i} y\right)+50 \phi\left(o, 2^{i} y\right)}{16^{i}} \\
& \quad(y \in \mathbb{R}, n \in \mathbb{N}) .
\end{aligned}
$$

Next, we show that $\left\{f\left(2^{n} y\right) / 16^{n}\right\}$ is a Cauchy sequence. For $m, n \in \mathbb{N}$, we have

$$
\begin{aligned}
& \left|\frac{f\left(2^{n+m} y\right)}{16^{n+m}}-\frac{f\left(2^{m} y\right)}{16^{m}}\right|=\frac{1}{16^{m}}\left|\frac{1\left(2^{n} 2^{m} y\right)}{16^{n}}-f\left(2^{m} y\right)\right| \\
& \leq \frac{1}{5376} \sum_{i=0}^{\infty} \frac{1}{16^{i+m}}\left(2 \phi\left(3\left(2^{i+m} y\right), 2^{i+m} y\right)+2 \phi\left(2\left(2^{i+m} y\right), 2^{i+m} y\right)\right. \\
& \left.\quad+\phi\left(0,2\left(2^{i+m} y\right)\right)+30 \phi\left(2^{i+m} y, 2^{i+m} y\right)+50 \phi\left(0,2^{i+m} y\right)\right) \\
& \rightarrow 0 \quad(n \rightarrow \infty),
\end{aligned}
$$

i.e., the sequence $\left\{f\left(2^{n} y\right) / 16^{n}\right\}$ is a Cauchy sequence. Since $\mathbb{R}$ is complete, the limit function $T(y)=\lim _{n \rightarrow \infty} f\left(2^{n} y\right) / 16^{n}$ exists for all $y \in \mathbb{R}$. From (3.14), we obtain

$$
\begin{aligned}
& |f(y)-T(y)|=\left|\lim _{n \rightarrow \infty} \frac{f\left(2^{n} y\right)}{16^{n}}-f(y)\right| \\
& \leq \frac{1}{5376} \sum_{i=0}^{\infty} \frac{2 \phi\left(3\left(2^{i} y\right), 2^{i} y\right)+2 \phi\left(2\left(2^{i} y\right), 2^{i} y\right)+\phi\left(0,2\left(2^{i} y\right)\right)+30 \phi\left(2^{i} y, 2^{i} y\right)+50 \phi\left(o, 2^{i} y\right)}{16^{i}} .
\end{aligned}
$$

Next, we show that $T$ satisfies the equation (1.9). Consider

$$
\begin{aligned}
D_{T}(x, y)= & T(x+3 y)+T(x-3 y)+T(x+2 y)+T(x-2 y)+22 T(x) \\
& -13 T(x+y)-13 T(x-y)-168 T(y) \\
= & \lim _{n \rightarrow \infty} \frac{1}{16^{n}}\left(f\left(2^{n}(x+3 y)\right)+f\left(2^{n}(x-3 y)\right)+f\left(2^{n}(x+2 y)\right)\right. \\
& \quad+f\left(2^{n}(x-2 y)\right)+22 f\left(2^{n} x\right)-13 f\left(2^{n}(x+y)\right)-13 f\left(2^{n}(x-y)\right) \\
& \left.\quad-168 f\left(2^{n} y\right)\right) \\
= & \lim _{n \rightarrow \infty} \frac{D_{f}\left(2^{n} x, 2^{n} y\right)}{16^{n}} .
\end{aligned}
$$


Thus,

$$
\left|D_{T}(x, y)\right| \leq \lim _{n \rightarrow \infty} \frac{1}{16^{n}}\left|D_{f}\left(2^{n} x, 2^{n} y\right)\right| \leq \lim _{n \rightarrow \infty} \frac{\phi\left(2^{n} x, 2^{n} y\right)}{16^{n}}=0 \quad(x, y \in \mathbb{R}),
$$

i.e., $T$ satisfies (1.9). To prove the uniqueness of $T$, suppose that there exists $S: \mathbb{R} \rightarrow \mathbb{R}$ satisfying (1.9) and (1.14). Lemma 3.1 gives

$$
\begin{aligned}
|T(y)-S(y)| & =\left|\frac{T\left(2^{n} y\right)}{16^{n}}-\frac{S\left(2^{n} y\right)}{16^{n}}\right| \\
\leq & \frac{1}{16^{n}}\left|T\left(2^{n} y\right)-f\left(2^{n} y\right)\right|+\frac{1}{16^{n}}\left|f\left(2^{n} y\right)-S\left(2^{n} y\right)\right| \\
\leq & \frac{2}{5376} \sum_{i=0}^{\infty} \frac{1}{16^{i+n}}\left(2 \phi\left(3\left(2^{i+n} y\right), 2^{i+n} y\right)+2 \phi\left(2\left(2^{i+n} y\right), 2^{i+n} y\right)\right. \\
& \left.\quad+\phi\left(0,2\left(2^{i+n} y\right)\right)+30 \phi\left(2^{i+n} y, 2^{i+n} y\right)+50 \phi\left(0,2^{i+n} y\right)\right) \\
& \rightarrow 0 \quad(n \rightarrow \infty),
\end{aligned}
$$

which completes the proof.

It is worth remarking that with a much longer proof the conditions that $f$ is even and $f(0)=0$ can be omitted.

Corollary 3.2. Let $\varepsilon>0$. If $f: \mathbb{R} \rightarrow \mathbb{R}$ is even with $f(0)=0$ and satisfies $\left|D_{f}(x, y)\right| \leq \varepsilon$, then there exists a unique $T: \mathbb{R} \rightarrow \mathbb{R}$ such that $T$ satisfies (1.9) and

$$
|f(y)-T(y)| \leq \frac{17 \varepsilon}{1008} \quad(y \in \mathbb{R}) .
$$

Proof. Taking $\phi(x, y)=\varepsilon$, the condition (1.12) holds and Theorem (1.3) II shows that there exists a unique $T: \mathbb{R} \rightarrow \mathbb{R}$ satisfying (1.9) and

$$
|f(y)-T(y)| \leq \frac{1}{5376} \sum_{i=0}^{\infty} \frac{2 \varepsilon+2 \varepsilon+\varepsilon+30 \varepsilon+50 \varepsilon}{16^{i}}=\frac{17 \varepsilon}{1008} .
$$

\section{Acknowledgments}

This research is supported by the Centre of Excellence in Mathematics, CHE, and Thammasat reseacch fund, Thailand. 


\section{References}

[1] J. Aczél, Lectures on Functional Equations and their Applications, Academic Press, New York (1966).

[2] J.K. Chung, P.K. Sahoo, On the general solution of a quartic functional equation, Bull. Korean Math. Soc., 40, No. 4 (2003), 565-576.

[3] S. Czerwik, Functional Equations and Inequalities in Several Variables, World Scientific, Singapore (2001).

[4] M. Kuczma, An Introduction to the Theory of Functional Equations and Inequalities, Państwowe Wydawnictwo Naukowe-Uniwersylet Ślaski, Warszawa-Kraków-Katowice (1985).

[5] P.K. Sahoo, On a functional equation characterizing polynomials of degree three, Bulletin of the Institute of Mathematics, Academia Sinica, 32 (2004), 35-44.

[6] P.K. Sahoo, A generalized cubic functional equation, Acta Mathematica Sinica, English Series Oct., 21, No. 5 (2005), 1159-1166. 